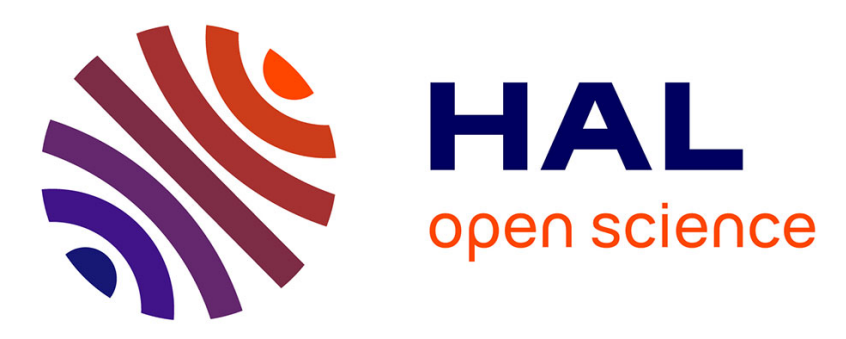

\title{
Whistler Waves Emitted by a Thin Modulated Electron Beam
}

\author{
A. Volokitin, C. Krafft, G. Matthieussent
}

\section{To cite this version:}

A. Volokitin, C. Krafft, G. Matthieussent. Whistler Waves Emitted by a Thin Modulated Electron Beam. Journal de Physique IV Proceedings, 1995, 05 (C6), pp.C6-79-C6-83. 10.1051/jp4:1995615 . jpa-00253978

\section{HAL Id: jpa-00253978 https://hal.science/jpa-00253978}

Submitted on 1 Jan 1995

HAL is a multi-disciplinary open access archive for the deposit and dissemination of scientific research documents, whether they are published or not. The documents may come from teaching and research institutions in France or abroad, or from public or private research centers.
L'archive ouverte pluridisciplinaire HAL, est destinée au dépôt et à la diffusion de documents scientifiques de niveau recherche, publiés ou non, émanant des établissements d'enseignement et de recherche français ou étrangers, des laboratoires publics ou privés. 


\title{
Whistler Waves Emitted by a Thin Modulated Electron Beam
}

\author{
A. Volokitin, C. Krafft* and G. Matthieussent* \\ Institute of Terrestrial Magnetism, Ionosphere and Radiowave Propagation, Academy of Sciences, \\ Troitsk, Moscow region, 142092, Russia \\ * Laboratoire de Physique des Gaz et des Plasmas, Université Paris Sud, Centre National \\ de la Recherche Scientifique, 91405 Orsay cedex, France
}

\begin{abstract}
The theory of whistler wave interaction with a modulated electron beam of finite radius, injected parallel to the magnetic field in an unbounded space plasma is considered. The study is done in the case of sheared whistlers interacting with the thin beam, when the parallel wave number is very small compared to the perpendicular one. The electromagnetic fields inside and outside the beam are determined in the linear approach; their asymptotic expressions in the beam vicinity are obtained in a rather simple form. The energy balance between the thin modulated beam and the sheared whistler is considered and dependence of emission power on the beam parameters is studied.
\end{abstract}

\section{INTRODUCTION}

Whistler waves excitation by electron beam injection has been observed in several space experiments [1-4]. Recent experimental results were also obtained on the Cherenkov emission of whistlers produced in the interaction of a modulated electron beam with a magnetized laboratory plasma in the Orsay experiment [5-6] and in the APEX space active experiment [7]. The existing theories of whistler wave interaction with a modulated electron beam of finite radius [8-10] did not take into account the beam evolution during this interaction. The purpose of our work is to provide a detailed study of the whistler features and to develop an approach which will be necessary in the forthcoming studies of the nonlinear interaction of an electron beam with a quasi-monochromatic whistler wave.

We consider the simple but very interesting case of a thin beam which has a radius $r_{b}$ smaller than the perpendicular wavelength of the whistler. Whistler waves are supposed to be sheared, that is $k_{z}=$ $k_{\|} \ll k_{\perp}$ (the $z$ axis is directed along the magnetic field $\mathbf{B}_{0}=B_{0} \mathbf{z}$ ) and the wave frequency $\omega$ is supposed to verify $\omega_{i} \ll \omega \ll \omega_{c}<\omega_{p}$, where $\omega_{i}$ and $\omega_{c}=e B_{0} / m c$ are respectively the ion and electron gyrofrequencies and $\omega_{p}^{2}=4 \pi n e^{2} / m$ is the electron plasma frequency (CGS units are used); $n$ is the plasma electron density, $e$ and $m$ are the electron charge and mass.

\section{SHEARED WHISTLERS}

The electromagnetic wave field $(\mathbf{E}, \mathbf{B})$ is described with the help of the vector and scalar potentials $\mathbf{A}$ and $\varphi$ as $\mathbf{B}=\nabla \times \mathbf{A}$ and $\mathbf{E}=-\nabla \varphi-\partial \mathbf{A} / c \partial t$. In our approximation of sheared whistlers, the perpendicular component of $\mathbf{A}$ is small and can be expressed in terms of the parallel vector potential $A_{z}$. The first equation for the wave potentials $A_{z}$ and $\varphi$ in the presence of a modulated beam current $\mathbf{j}_{\mathbf{b}}=\left(j_{b z}, \mathbf{j}_{b \perp}\right)$ follows from the equation of electron motion and takes into account that the parallel electric current in the whistler wave is supported mainly by electrons. The second whistler wave equation follows from the quasi-neutrality condition $\boldsymbol{\nabla} \cdot \mathbf{j}=0$, where $\mathbf{j}=\left(j_{\mathbf{z}}, j_{\perp}\right)$ is the total current, taking into account the $(\mathbf{E} \times \mathbf{B})$ and polarization drifts of electrons. In the approximation $A_{z} \gg A_{\perp}$, which is valid for sheared whistlers with $k_{z} \ll k_{\perp}$ and $\omega \ll \omega_{c}\left(c k / \omega_{p}\right)^{2}$, one obtains the wave equations

$$
\begin{gathered}
\frac{\partial}{\partial t}\left(1-\frac{c^{2} \nabla^{2}}{\omega_{p}^{2}}\right) A_{z}+c \frac{\partial}{\partial z} \varphi=\frac{c}{\omega_{p}^{2}} \frac{\partial}{\partial t} 4 \pi j_{b z} \\
\frac{\omega_{p}^{2}}{\omega_{c}^{2}}\left(\nabla_{\perp}^{2}-\frac{\omega_{p}^{2}}{c^{2}}\right) \frac{\partial}{\partial t} \varphi+\omega_{p i}^{2} \nabla_{\perp}^{2} \int^{t} d t \varphi+c \frac{\partial}{\partial z} \nabla^{2} A_{z}=-\frac{\omega_{p}^{2}}{\omega_{c}} \frac{4 \pi}{c^{2}} \nabla^{-2} \frac{\partial}{\partial t}\left(\nabla \times \mathbf{j}_{\mathbf{b} \perp}\right) \cdot \mathbf{z}
\end{gathered}
$$




$$
\nabla^{2} \mathbf{A}_{\perp} \simeq \frac{\omega_{p}^{2}}{c \omega_{c}} \mathbf{z} \times \nabla \varphi-\frac{4 \pi}{c} \mathbf{j}_{\mathbf{b} \perp}
$$

where $\omega_{p i}$ is the ion plasma frequency.

The equations (1) determine the wave energy balance in a cylindrical volume

$$
\frac{\partial}{\partial t} W+\frac{\partial}{\partial z} \mathcal{P}_{z}+\mathcal{P}_{r}=\mathcal{R}
$$

where $W, \mathcal{P}_{z}$ and $\mathcal{R}$ are the longitudinal energy density, the density of longitudinal energy flux and the work of the beam on the whistler. The radial energy losses per unit length $\mathcal{P}_{r}$ are given by

$$
\mathcal{P}_{r}=-\int_{0}^{2 \pi} \frac{d \theta}{4 \pi} \mathbf{r}\left(\frac{\omega_{p}^{2}}{\omega_{c}^{2}} \varphi \nabla_{\perp} \frac{\partial \varphi}{\partial t}+\omega_{p i}^{2} \varphi \nabla_{\perp} \int^{t} \varphi d t+c \varphi \nabla_{\perp} \frac{\partial A_{z}}{\partial z}+\frac{c^{2}}{\omega_{p}^{2}} \frac{\partial \nabla_{\perp}^{2} A_{z}}{\partial t} \nabla_{\perp} A_{z}\right)
$$

We suppose that it is possible to avoid processes which could modify the beam structure and the beam electron distribution function. We consider two cases: (i) the parallel beam injection $\mathbf{j}_{b}=\mathbf{j}(r) \cos (\omega(t-$ $\left.\left.z / v_{b z}\right)\right)$; (ii) the injection at a finite pitch angle with respect to the magnetic field, when the beam forms a spiral structure of current $\mathbf{j}_{b}=\left(j_{b z}, j_{b \theta}\right)$, with $j_{b \theta}=-e \pi r_{b}^{2} n_{b} \cos \left(\omega\left(t-z / v_{b z}\right)\right)\left(v_{b \perp} / R\right) \delta\left(\theta-\omega_{c} t\right) \delta(r-R)$, where $\mathbf{v}_{b}=\left(v_{b z}, v_{b \perp}\right)$ is the beam velocity and $R=v_{b \perp} / \omega_{c}$ is the Larmor radius; $n_{b}$ is the beam density. In both cases the solutions of (1) with boundary conditions proper for waves going out to infinity have the form of Bessel integrals: $\varphi=\varphi(r) e^{-i\left(\omega t-k_{z} z\right)}+$ c.c. with $\varphi(r)=\sum_{n} \int_{0}^{\infty} k d k J_{n}(k r) e^{i n \theta} \varphi_{k n}$ and $A_{z}(r)=\sum_{n} \int_{0}^{\infty} k d k J_{n}(k r) e^{i n \theta} A_{z, k n}$, where

$$
\begin{gathered}
\varphi_{k n}=\frac{4 \pi}{\epsilon(k, \omega)}\left\{\frac{\left(k_{z} j_{b z, k n} / \omega\right)}{\left(k^{2}+\omega_{p}^{2} / c^{2}\right)}-\frac{\omega_{p}^{2}}{c^{2} k^{4}} \frac{\left(\boldsymbol{\nabla} \times \mathbf{j}_{\mathbf{b} \perp, \mathbf{k n}}\right) \cdot \mathbf{z}}{\omega_{c}}\right\} \\
A_{z, k n}=\frac{\frac{c k_{z}}{\omega}}{\left(1+c^{2} k^{2} / \omega_{p}^{2}\right)} \frac{4 \pi}{\epsilon(k, \omega)}\left\{\frac{\left(k_{z} j_{b z, k n} / \omega\right)}{\left(k^{2}+\omega_{p}^{2} / c^{2}\right)}-\frac{\omega_{p}^{2}}{c^{2} k^{4}} \frac{\left(\boldsymbol{\nabla} \times \mathbf{j}_{\mathbf{b} \perp, \mathbf{k n}}\right) \cdot \mathbf{z}}{\omega_{c}}\right\}+\frac{4 \pi c j_{b z, k n}}{\omega_{p}^{2}\left(1+c^{2} k^{2} / \omega_{p}^{2}\right)} \\
\epsilon(k, \omega)=\frac{\omega_{p}^{2}}{\omega_{c}^{2}}\left(1+\frac{\omega_{p}^{2}}{c^{2} k^{2}}\right)-\frac{\omega_{p i}^{2}}{\omega^{2}}-\frac{c^{2} k_{z}^{2}}{\omega^{2}}\left(1+\frac{c^{2} k^{2}}{\omega_{p}^{2}}\right)^{-1}
\end{gathered}
$$

$\epsilon(k, \omega)$ is the effective "electromagnetic" dielectric constant and $\left(k_{z} j_{b z, k n} / \omega\right)=\int^{t} d t\left(\partial \rho_{b z, k n} / \partial t\right)$ is the beam electric charge density.

\section{PARALLEL INJECTION WITH RESPECT TO THE MAGNETIC FIELD}

In the parallel injection case, one has $k_{\perp} r_{b} \ll 1$ and it is possible to neglect the internal structure of the beam and the perpendicular current $\dot{j}_{\mathrm{b} \perp}$. At the beam density $n_{b}$ (constant for $r<r_{b}$ ), one has $\left(k_{z} j_{b z} / \omega\right) \simeq \rho_{b}\left(J_{1}\left(k r_{b}\right) / k r_{b}\right)$, where $\rho_{b}=-\left(e n_{b}\left(k_{z} v_{b} / \omega\right) r_{b}^{2} / 2 \pi\right)$ is the beam charge density per unit length. To calculate the wave field we transform the integral $\varphi(r)=\int_{0}^{\infty} k d k J_{0}(k r) \varphi_{k}$ on the total axis $k$ using the presentation of Bessel functions in terms of Hankel functions. Thus we obtain

$$
\varphi(r)=\frac{1}{2} \oint_{C} d k \frac{4 \pi \rho_{b} / r_{b}}{\epsilon(k, \omega)\left(k^{2}+\omega_{p}^{2} / c^{2}\right)}\left\{\begin{array}{l}
J_{1}\left(k r_{b}\right) H_{0}^{(1)}(k r) \\
J_{0}(k r) H_{1}^{(1)}\left(k r_{b}\right)
\end{array}\right\} \begin{aligned}
& r>r_{b} \\
& r<r_{b}
\end{aligned}
$$

The integral path has been transformed in the closed contour $C$ which passes above the real negative axis and below the real positive axis, follows an infinitesimal semicircle around the origin and is closed by a semicircle at infinity in the upper half plane. The main contribution to (5) comes from the two poles of $\epsilon(k, \omega)=0$ : their positions $\left(k_{1}\right)$ and $\left(-k_{2}\right)$ are given by $c^{2} k^{2} / \omega_{p}^{2}=\left[\mathcal{B} \pm \sqrt{\mathcal{B}^{2}-4 \mathcal{A}}\right] / 2$, where $\mathcal{B}=\omega_{c}^{2} c^{2} / \omega_{p}^{2} v_{b}^{2}+\omega_{l h}^{2} / \omega^{2}-2$ and $\mathcal{A}=1-\omega_{l h}^{2} / \omega^{2}$ ( $\omega_{l h}$ is the lower hybrid frequency). The poles $k_{1}$ and $k_{2}$ are merging at $v_{b}=c \omega_{c} / 2 \omega_{p}$, where the group velocity $v_{g \perp}$ vanishes (double pole case).

Let us introduce a typical scale for the potential of the beam, as $\Phi=-\left(\omega_{c} / \omega_{p}\right)^{2} e \pi r_{b}^{2} n_{b}\left(1-\omega_{l h}^{2} / \omega^{2}\right)^{-1}$; after integration we obtain the expressions of both potentials of whistler waves as 


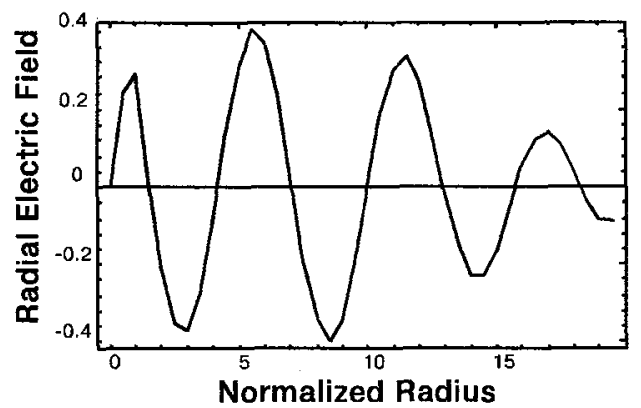

Figure 1: Real component of the normalized radial electric field as a function of the normalized distance from the center of the beam.

$$
\begin{gathered}
A_{z}(r)=\frac{i \Phi \frac{c k_{z}}{\omega} \frac{\omega_{p}^{2}}{c^{2}}}{\left(k_{1}^{2}-k_{2}^{2}\right)}\left\{\frac{k_{2}^{2} J_{1}\left(k_{2} r_{b}\right) H_{0}^{(2)}\left(k_{2} r\right)}{k_{2} r_{b}\left(\frac{\omega_{p}^{2}}{c^{2}}+k_{2}^{2}\right)}+\frac{k_{1}^{2} J_{1}\left(k_{1} r_{b}\right) H_{0}^{(1)}\left(k_{1} r\right)}{k_{1} r_{b}\left(\frac{\omega_{p}^{2}}{c^{2}}+k_{1}^{2}\right)}+\frac{\frac{i \omega_{p}}{c} J_{1}\left(\frac{i \omega_{p}}{c} r_{b}\right) H_{0}^{(1)}\left(\frac{i \omega_{p}}{c} r\right)\left(k_{1}^{2}-k_{2}^{2}\right)}{r_{b}\left(\omega_{p}^{2} / c^{2}+k_{2}^{2}\right)\left(\frac{\omega_{p}^{2}}{c^{2}}+k_{1}^{2}\right)}\right\} \\
\varphi(r)=\frac{i \Phi}{\left(k_{1}^{2}-k_{2}^{2}\right)} \cdot\left\{k_{2}^{2} \frac{J_{1}\left(k_{2} r_{b}\right)}{k_{2} r_{b}} H_{0}^{(2)}\left(k_{2} r\right)+k_{1}^{2} \frac{J_{1}\left(k_{1} r_{b}\right)}{k_{1} r_{b}} H_{0}^{(1)}\left(k_{1} r\right)\right\} \quad r>r_{b} \\
A_{z}(r)=\frac{i \Phi \frac{c k_{z}}{\omega} \frac{\omega_{p}^{2}}{c^{2}}}{\left(k_{1}^{2}-k_{2}^{2}\right)}\left\{\frac{k_{2}^{2} J_{0}\left(k_{2} r\right) H_{1}^{(2)}\left(k_{2} r_{b}\right)}{k_{2} r_{b}\left(\frac{\omega_{p}^{2}}{c^{2}}+k_{2}^{2}\right)}+\frac{k_{1}^{2} J_{0}\left(k_{1} r\right) H_{1}^{(1)}\left(k_{1} r_{b}\right)}{k_{1} r_{b}\left(\frac{\omega_{p}^{2}}{c^{2}}+k_{1}^{2}\right)}+\frac{\left(k_{1}^{2}-k_{2}^{2}\right) \frac{i \omega_{p}}{c} J_{0}\left(\frac{i \omega_{p}}{c} r\right) H_{1}^{(1)}\left(\frac{i \omega_{p}}{c} r_{b}\right)}{r_{b}\left(\frac{\omega_{p}^{2}}{c^{2}}+k_{2}^{2}\right)\left(\frac{\omega_{p}^{2}}{c^{2}}+k_{1}^{2}\right)}\right\} \\
\left.\varphi(r)=\frac{i \Phi}{\left(k_{1}^{2}-k_{2}^{2}\right)} \cdot\left\{k_{2}^{2} \frac{H_{1}^{(2)}\left(k_{2} r_{b}\right)}{k_{2} r_{b}} J_{0}\left(k_{2} r\right)+k_{1}^{2} \frac{H_{1}^{(1)}\left(k_{1} r_{b}\right)}{k_{1} r_{b}} J_{0}\left(k_{1} r\right)\right\} \quad r<r_{b}\right)
\end{gathered}
$$

The two resonant waves $\left(k_{1}\right.$ and $\left.-k_{2}\right)$ are emitted coherently; so, the interference between them is strong near the beam. With the help of the asymptotic developments of the Hankel functions [11] at $k r \rightarrow 0$, the expressions for the potentials in the vicinity of the beam $\left(r_{b}<r \ll k^{-1}\right)$ can be obtained. As an example, let us present the expression of the radial electric field in the vicinity of the double pole $\left(k_{1}^{2} \simeq k_{2}^{2}\right): E_{r}(r) \simeq(\Phi / \pi r)\left(1+\pi(-1+i)\left(r \omega_{p} / 2 c\right)^{2}\left(\nu \omega_{c}^{2} / \omega^{3}\right)^{-1 / 2}\right)$, where $\nu$ is the dissipation frequency. Thus, if $c / \omega_{p}>r_{b}>\left(c / \omega_{p}\right)\left(\nu \omega_{c}^{2} / 2 \omega^{3}\right)^{1 / 4}$, the electric field maximum is far from the beam (at $r \simeq c / \omega_{p}$ ). The wave energy goes to infinity when $\nu$ tends to zero. It means that the time required to achieve this state is infinite and it is necessary to consider the beam evolution and the corresponding nonlinear processes.

The potentials and the fields have been computed numerically. Fig. 1 shows the real component of the normalized radial electric field $\widetilde{E}(\widetilde{r})=-\partial \widetilde{\varphi} / \partial \widetilde{r} ; \widetilde{r}=r \omega_{p} / c$ is the dimensionless distance from the center of the beam and $\tilde{\varphi}=\varphi / \Phi$ is the normalized potential.

\section{SPIRAL BEAM: INJECTION OBLIQUE TO THE MAGNETIC FIELD}

For the spiral electron beam, the source terms are $\left[\left(\nabla \times \mathbf{j}_{\mathrm{b} \perp}\right) \cdot \mathbf{z}\right]_{k n}=(2 \pi)^{-2} e \pi r_{b}^{2} n_{b} v_{b \perp} e^{-i n \omega_{c} t} \partial J_{n}(k R) / \partial R$ and $\rho_{k n}=-(2 \pi)^{-2} e \pi r_{b}^{2} n_{b}\left(k_{z} v_{b z} / \omega\right) e^{-i n \omega_{c} t} J_{n}(k R)$; then the potentials for sheared whistlers (4) are

$$
\begin{gathered}
\varphi_{k n}=-\frac{v_{b \perp} e r_{b}^{2} n_{b}}{\epsilon(k, \omega)}\left\{\frac{k_{z} v_{b z}}{v_{b \perp} \omega} \frac{J_{n}(k R)}{\left(k^{2}+\omega_{p}^{2} / c^{2}\right)}-\frac{\omega_{p}^{2}}{c^{2} k^{4}} \frac{\partial J_{n}(k R) / \partial R}{\omega_{c}}\right\} \\
A_{z, k n}=-\frac{c k_{z}}{\omega\left(1+c^{2} k^{2} / \omega_{p}^{2}\right)} \frac{v_{b \perp} e r_{b}^{2} n_{b}}{\epsilon(k, \omega)}\left\{\frac{k_{z} v_{b z}}{v_{b \perp} \omega} \frac{J_{n}(k R)}{\left(k^{2}+\omega_{p}^{2} / c^{2}\right)}-\frac{\omega_{p}^{2}}{c^{2} k^{4}} \frac{\partial J_{n}(k R) / \partial R}{\omega_{c}}\right\}
\end{gathered}
$$

The integral path in the potential $\varphi(r, \theta)=\sum_{n} \int_{0}^{\infty} k d k J_{n}(k r) e^{i n \theta} \varphi_{k n}=\sum_{n} I_{n} e^{i n \theta}$ is transformed in the closed contour $C$ and $J_{n}$ can be expressed by Hankel functions. The calculation of $\sum_{n} I_{n} e^{i n \theta}$ is done using 
the relation [11] $e^{i s \psi} H_{s}^{(1,2)}(k \rho)=\sum_{n=-\infty}^{\infty} J_{n}(k R) H_{n+s}^{(1,2)}(k r) e^{i n \theta}$, where $\rho=\sqrt{r^{2}+R^{2}-2 r R \cos \theta} \neq 0$ $(r \neq R), e^{i 2 \psi}=\left(r-R e^{-i \theta}\right) /\left(r-R e^{i \theta}\right)$ (for $r>R$ ), and $s$ is an integer number. Noticing that $\cos \psi=(r-R \cos \theta) / \rho$, one obtains the potentials

$$
\begin{gathered}
\frac{\varphi(r, \theta)}{\Phi}=\frac{i}{2}\left\{\frac{k_{z} v_{b z}}{\omega} \mathcal{F}_{0}\left(k_{1}, k_{2}, r, \theta\right)+\frac{(R-r \cos \theta)}{\rho}\left(\mathcal{F}_{1}\left(k_{1}, k_{2}, r, \theta\right)-i \frac{2}{\pi} \frac{\omega_{p}^{4}}{c^{4}} \frac{R}{k_{1}^{2} k_{2}^{2} \rho}\right)\right\} \\
\mathcal{F}_{0}\left(k_{1}, k_{2}, r, \theta\right)=\frac{k_{1}^{2} H_{0}^{(1)}\left(k_{1} \rho\right)+k_{2}^{2} H_{0}^{(2)}\left(k_{2} \rho\right)}{\left(k_{1}^{2}-k_{2}^{2}\right)} \\
\mathcal{F}_{1}\left(k_{1}, k_{2}, r, \theta\right)=\frac{R \omega_{p}^{2}}{c^{2}} \frac{\left(k_{1}\left(1+\omega_{p}^{2} / k_{1}^{2} c^{2}\right) H_{1}^{(1)}\left(k_{1} \rho\right)+k_{2}\left(1+\omega_{p}^{2} / k_{2}^{2} c^{2}\right) H_{1}^{(2)}\left(k_{2} \rho\right)\right)}{\left(k_{1}^{2}-k_{2}^{2}\right)} \\
\frac{\omega}{k_{z} c} \frac{A_{z}(r, \theta)}{\Phi}=\frac{i}{2}\left\{\frac{k_{z} v_{b z}}{\omega} \mathcal{G}_{0}\left(k_{1}, k_{2}, r, \theta\right)+\frac{(R-r \cos \theta)}{\rho}\left(\mathcal{G}_{1}\left(k_{1}, k_{2}, r, \theta\right)-i \frac{2}{\pi} \frac{\omega_{p}^{4}}{c^{4}} \frac{R}{k_{1}^{2} k_{2}^{2} \rho}\right)\right\} \\
\mathcal{G}_{0}\left(k_{1}, k_{2}, r, \theta\right)=\frac{\omega_{p}^{2}}{\left(k_{1}^{2}-k_{2}^{2}\right) c^{2}}\left(\frac{H_{0}^{(1)}\left(k_{1} \rho\right)}{1+\omega_{p}^{2} / k_{1}^{2} c^{2}}+\frac{H_{0}^{(2)}\left(k_{2} \rho\right)}{1+\omega_{p}^{2} / k_{2}^{2} c^{2}}\right)-\frac{\omega_{p}^{4}}{c^{4}} \frac{H_{0}^{(1)}\left(i \omega_{p} \rho / c\right)}{\left(k_{1}^{2}+\omega_{p}^{2} / c^{2}\right)\left(k_{2}^{2}+\omega_{p}^{2} / c^{2}\right)} \\
\mathcal{G}_{1}\left(k_{1}, k_{2}, r, \theta\right)=\frac{\omega_{p}^{4} R^{2}}{\left(k_{1}^{2}-k_{2}^{2}\right) c^{4}}\left(\frac{H_{1}^{(1)}\left(k_{1} \rho\right)}{R k_{1}}+\frac{H_{1}^{(2)}\left(k_{2} \rho\right)}{R k_{2}}\right)
\end{gathered}
$$

The same expressions give the potentials in the internal zone of the beam $(r<R)$. The potential (8) has a singularity at $\rho=0(r=R)$, along the trajectory of the beam electrons. This singularity has a rather slow variation - logarithmic - and disappears for a finite thickness of the tube described by the beam electrons. The vector potential and the components $E_{\theta}, B_{r}$ and $B_{\theta}$ are finite at $r=R$.

Figure 2 shows the variation of the azimuthal magnetic field (real and imaginary components) as a function of the azimuthal angle $\theta$ and the normalized distance $\widetilde{r}$ from the center of the beam.
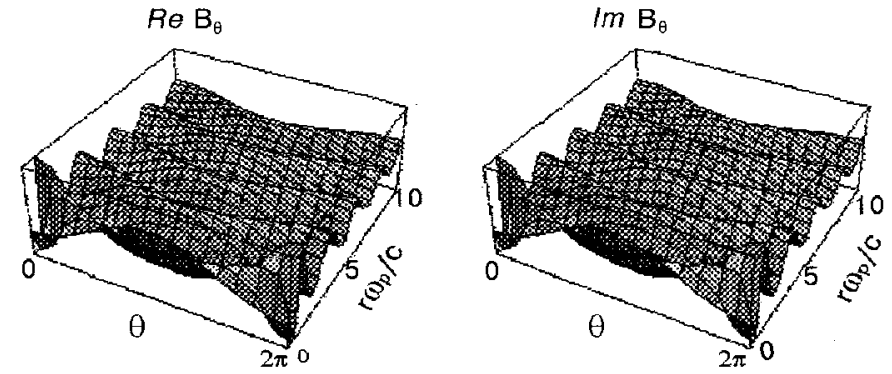

Figure 2: Azimuthal magnetic field (real and imaginary components) as a function of the azimuthal angle $\theta$ and the normalized distance $\widetilde{r}$ from the center of the beam.

\section{EMISSION RATE}

It is necessary to be careful with the estimation of the energy flux along the magnetic field. In the considered steady state regime, which corresponds to an infinite time of beam injection, the longitudinal energy flux is infinite. To avoid this problem and to obtain the beam energy losses due to wave radiation, we consider the transverse energy flux. We calculate the radial energy flux per unit length according to the Poynting vector (3) and using the asymptotic expressions of the potentials in the far wave zone as well as the dispersion equation and the perpendicular group velocity $v_{g \perp}=\partial \omega / \partial k_{\perp}$. The interference terms between two resonant waves $\left(k_{1}\right.$ and $\left.-k_{2}\right)$ are strong near the beam, but at large distances their coherence can be destroyed by various effects as wave damping and beam dynamics. Neglecting these terms we obtain

$$
\mathcal{P}_{r}=\frac{\Phi^{2}}{2 \pi} \frac{\omega_{p}^{2}}{\omega_{c}^{2}} \sum_{i=1}^{2} \frac{\left|v_{g \perp i}\right| k_{i}^{5}}{\left[\left(k_{1}^{2}-k_{2}^{2}\right)\right]^{2}}\left[\left(\frac{k_{z} v_{b z}}{\omega}\right)^{2}+\frac{1}{2}\left(\frac{\omega_{p} R}{c}\right)^{2}\left(\frac{\omega_{p}}{k_{i} c}\left(\frac{\omega_{p}^{2}}{k_{i}^{2} c^{2}}+1\right)\right)^{2}\right]\left[1+\frac{\omega_{p}^{2}}{k_{i}^{2} c^{2}}\right]
$$




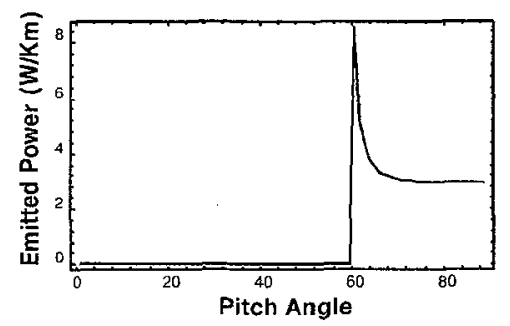

Figure 3: Radial energy loss by unit length as a function of the pitch angle of injection at a fixed electric beam current.

Noticing that potentials in the far wave zone for parallel injection are different from those of the spiral beam case only by terms proportional to $R$, formula (9) is applicable to the parallel injection case if one puts $R=0$. The total flux can be calculated taking into account that at large distances, the component of flux along the magnetic field is given by $P_{z}=P_{r}\left(v_{g z} / v_{g \perp}\right)$.

The radial energy loss per unit length has been calculated numerically according (10) as a function of the beam velocity and the injection pitch angle at a fixed electric current $I_{b}$. Results presented on Fig. 3 for the Cherenkov resonance show a strong emission increasing in the vicinity of the double pole.

\section{Acknowledgments}

A.S. Volokitin acknowledges the financial support from DRET and CNRS durind his visit to the Laboratoire de Physique des Gaz et des Plasmas, University Paris-South, Orsay.

\section{References}

[1] Bush I. et al., Am. Geophys. 36, (1980) 341-350.

2] Lavergnat J., The French-Soviet Experiment ARAKS: Main results, Artificial Particle Beams in Space Plasma Studies, (Plenum, New York, 1982), pp. 87-94.

[3] Farrell W.M., Gurnett D.A. and Goertz C.K., J. Geophys. Res. 94, (1989) 443-456.

4] Neubert T. et al., Adv Space Res. 36, (1990) 469-485

5 Krafft C. et al., Phys. Revi Lett. 72, (1994) 649-652.

6. Krafft C. et al., Phys. Plasmas 1(7), (1994) 2163-2171

7] Oraevsky V.N. et al., J. Atmos. Terr. Phys. 56, 3, (1994) 423-431

8. Lavergnat J. et al., P̉hys. Fluids 25, (1982) 1073-1082.

[9] Lavergnat J., Lehner T. and Matthieussent G., Phys. Fluids 27, (1984) 1632-1639.

10] Neubert T. and Harker K.L., Planet. Space Sci. 36, (1988) 469-490.

[11] Abramowitz M. and Stegun I.A., Handbook of Mathematical Functions, Dover Publications, New York, (1970). 